\title{
Caristi Type Selections of Multivalued Mappings
}

\author{
Calogero Vetro ${ }^{1}$ and Francesca Vetro ${ }^{2}$ \\ ${ }^{1}$ Dipartimento di Matematica e Informatica, Università degli Studi di Palermo, Via Archirafi 34, 90123 Palermo, Italy \\ ${ }^{2}$ Dipartimento Energia, Ingegneria dell'Informazione e Modelli Matematici (DEIM), Università degli Studi di Palermo, \\ Viale delle Scienze, 90128 Palermo, Italy
}

Correspondence should be addressed to Francesca Vetro; francesca.vetro@unipa.it

Received 29 October 2014; Accepted 16 December 2014

Academic Editor: Sompong Dhompongsa

Copyright (c) 2015 C. Vetro and F. Vetro. This is an open access article distributed under the Creative Commons Attribution License, which permits unrestricted use, distribution, and reproduction in any medium, provided the original work is properly cited.

Multivalued mappings and related selection theorems are fundamental tools in many branches of mathematics and applied sciences. In this paper we continue this theory and prove the existence of Caristi type selections for generalized multivalued contractions on complete metric spaces, by using some classes of functions. Also we prove fixed point and quasi-fixed point theorems.

\section{Introduction and Preliminaries}

In 1998, Repovs and Semenov [1] furnished a comprehensive study of the theory of continuous selections for multivalued mappings. They point out that "this interesting branch of modern topology was started by Michael [2] and, since then, has received a great amount of interest with various applications outside topology, for instance, approximation theory, control theory, convex sets, differential inclusions, economics, fixed point theory, and vector measures." Thus an interesting matter is to obtain existence conditions for selections, under different regularity hypotheses, for instance, Lipschitz-continuity and measurability; see also [3-5]. In particular, we are interested in developing this theory for fixed point theorems, by using Caristi's mappings. Some precise results concerning existence of fixed points for Caristi's single-valued and multivalued mappings and data dependence of fixed points set are proved in [6]; see also the references therein. We recall that Browder [7] was the first author to use continuous selections to prove a fixed point result; but the first result of Caristi type selection was proved by Jachymski [8] for Nadler's multivalued contraction with closed values.

Definition 1. Let $(X, d)$ be a metric space. A function $\phi: X \rightarrow$ $[0,+\infty)$ is lower semicontinuous at $x \in X$ if and only if, for every sequence $\left\{x_{n}\right\}$ in $X$ with $x_{n} \rightarrow x$ as $n \rightarrow+\infty$, $\liminf _{n \rightarrow+\infty} \phi\left(x_{n}\right) \geq \phi(x)$. Also, $\phi$ is lower semicontinuous if and only if it is lower semicontinuous at every $x \in X$.
Also, $L(y):=\{x \in X: \phi(x) \leq y\}$ is called the lower counter set defined by a point $y \in[0,+\infty)$. Then the following results hold true.

Proposition 2. Let $(X, d)$ be a metric space. Let $\phi: X \rightarrow$ $[0,+\infty)$ be a function. Then, $\phi$ is lower semicontinuous if and only if $L(y)$ is closed for every $y \in[0,+\infty)$.

Theorem 3 (Caristi [9]). Let $(X, d)$ be a complete metric space and let $f: X \rightarrow X$ be a mapping not necessarily continuous. Assume that there exists a function $\phi: X \rightarrow[0,+\infty)$, which is lower semicontinuous, such that

$$
d(x, f x) \leq \phi(x)-\phi(f x) \quad \forall x \in X
$$

Then, $f$ has a fixed point $z$; that is, $z=f z$.

Also, $f$ is called Caristi's mapping on $(X, d)$. On the other hand, Nadler $[10,11]$ established the following result.

Theorem 4 (Nadler $[10,11])$. Let $(X, d)$ be a complete metric space and let $F: X \rightarrow C l(X)$ be a multivalued mapping such that, for some $k \in(0,1)$, one has

$$
H(F x, F y) \leq k d(x, y) \quad \forall x, y \in X,
$$

where $\mathrm{Cl}(X)$ denotes the class of all nonempty closed subsets of $X$ and $H$ denotes a generalized Hausdorff metric on $\mathrm{Cl}(X)$. Then $F$ has a fixed point $z$; that is, $z \in F z$. 
For $A, B \in C l(X)$, we recall that $H(A, B)=\max \{\sup \{D(x$, $B): x \in A\}, \sup \{D(y, A): y \in B\}\}$, where $D(x, B)=\inf \{d(x$, $y): y \in B\}$. Also, a multivalued mapping $F$ satisfying the assumption of Theorem 4 is called Nadler's multivalued contraction.

Definition 5. Let $F: X \rightarrow C l(X)$ be a multivalued mapping and let $f: X \rightarrow X$ be a (single-valued) mapping. Then, $f$ is said to be a selection for $F$ if

$$
f x \in F x, \quad x \in X .
$$

Also $f$ is called Caristi type selection if it is Caristi's mapping. As mentioned above, Jachymski established existence theorems stating that certain multivalued mappings admit selections that are Caristi's mappings, which do not need to be continuous (see, for instance, Example 1 in [8]).

Theorem 6 (Jachymski [8]). If $F$ is Nadler's multivalued contraction on a complete metric space $(X, d)$, then $F$ admits a selection $f: X \rightarrow X$, which is Caristi's mapping on $(X, d)$ generated by a Lipschitz function $\phi$.

Clearly, Theorem 3 yields Theorem 4; that is, every Nadler's multivalued mapping admits a fixed point, but the converse does not hold in general. Obviously, if the multivalued mapping does not admit a fixed point, then a Caristi type selection cannot exist. The following example illustrates the case of a multivalued mapping which does not admit a Caristi type selection, even if it has a fixed point.

Example 7 (Xu [12]). Consider the complete metric space $([0,+\infty), d)$, where $d$ denotes the standard metric. Define $F$ : $[0,+\infty) \rightarrow C l([0,+\infty))$ as $F x=[2 x, 3 x]$ for all $x \in[0,+\infty)$.

Trivially, 0 is a unique fixed point of $F$. Now, assume that there exists a Caristi type selection for $F$, say $f$. Then, referring to notions and notations of Theorem 3, we write $d(x, f x) \leq \phi(x)-\phi(f x)$ for all $x \in[0,+\infty)$. By definition of $F$, we have $2 x \leq f x \leq 3 x$ and so $x \leq \phi(x)-\phi(f x)$ for all $x \in(0,+\infty)$. By iteration, we can get easily that

$$
f^{n} x \leq \phi\left(f^{n} x\right)-\phi\left(f^{n+1} x\right) \quad \forall n \in \mathbb{N} \cup\{0\} .
$$

This implies that the sequence $\left\{\phi\left(f^{n} x\right)\right\}$ is nonincreasing and so, being bounded below, convergent to some $r \geq 0$. Also, from (4), as $n \rightarrow+\infty$, we get $f^{n} x \rightarrow 0$. On the other hand, the reader can immediately prove that $\left\{f^{n} x\right\}$ is a strictly increasing sequence and hence we get a contradiction with the above limit. Then we conclude that $f$ is not a Caristi type selection.

Definition 8. Given a function $\mu:[0,+\infty) \rightarrow[0,+\infty)$ with $\mu(t)<t$ for $t>0$, a multivalued mapping $F: X \rightarrow C l(X)$ is said to be a multivalued $\mu$-contraction if

$$
H(F x, F y) \leq \mu(d(x, y)) \quad \forall x, y \in X .
$$

Definition 9. A function $\mu:[0,+\infty) \rightarrow[0,+\infty)$ is said to be subadditive if

$$
\mu(s+t) \leq \mu(s)+\mu(t) \quad \forall s, t \in[0,+\infty) .
$$

Also, $\mu$ is said to be superadditive if the reverse inequality holds true.

Theorem 10 (Jachymski [8]). Let $F: X \rightarrow C l(X)$ be a multivalued $\mu$-contraction on a complete metric space $(X, d)$ such that $\mu$ is superadditive, and the function $t \rightarrow t-\mu(t)(t \in$ $[0,+\infty))$ is nondecreasing. Then there exist a selection $f$ of $F$ and a function $h:[0,+\infty) \rightarrow[0,+\infty)$, which is nondecreasing and subadditive and continuous at $t=0$ such that $h^{-1}(\{0\})=\{0\}$. Moreover, there is an equivalent metric $\varrho$ such that $(X, \varrho)$ is complete and $f$ is Caristi's mapping on $(X, \varrho)$.

In this paper we continue this study and prove the existence of Caristi type selections for generalized multivalued contractions on complete metric spaces. Our results fit into the theory of selections for multivalued mappings showing certain ways to establish selection theorems, by using some classes of functions. Also we prove fixed point and quasi-fixed point theorems. We remark that the existence of a Caristi type selection for a multivalued mapping ensures the existence of a fixed point.

\section{Caristi Type Selection Theorems}

We start to develop our theory by using the concept of lower semicontinuity, which is one of the most important concepts in multivalued analysis.

Theorem 11. Let $(X, d)$ be a complete metric space. Let $F$ : $X \rightarrow 2^{X} \backslash\{\emptyset\}$ be a multivalued mapping and let $q>1$ be a real number. Consider $S_{q}(x):=\{y \in F x: d(x, y) \leq q D(x, F x)\}$ and suppose that $F$ satisfies the following conditions:

(i) there exist two nonnegative real numbers $a, b$ with $a q+$ $b<1$ such that, for each $x \in X$, there is $y \in S_{q}(x)$ having the property

$$
D(y, F y) \leq a d(x, y)+b D(x, F x)
$$

(ii) the function $p: X \rightarrow[0,+\infty)$ defined by $p x:=$ $D(x, F x)$, for all $x \in X$, is lower semicontinuous.

Then $F$ has a selection $f$ that is Caristi's mapping.

Proof. By the axiom of choice and condition (i), there is a mapping $f: X \rightarrow X$ with $f x \in S_{q}(x)$ for all $x \in X$ such that

$$
D(f x, F f x) \leq a d(x, f x)+b D(x, F x)
$$

and so

$$
D(f x, F f x)-b D(x, F x) \leq a d(x, f x) .
$$

Then

$$
\begin{aligned}
(1-b-a q) d(x, f x) \leq & (1-b) q D(x, F x)-a q d(x, f x) \\
\leq & (1-b) q D(x, F x)-q D(f x, F f x) \\
& +b q D(x, F x) \\
= & q D(x, F x)-q D(f x, F f x) .
\end{aligned}
$$


By condition (ii), the function $\phi: X \rightarrow[0,+\infty)$ defined by $\phi(t):=(q /(1-b-a q)) D(t, F t)$, for all $t \in X$, is lower semicontinuous and hence $f$ is Caristi's mapping that is a selection of $F$.

Example 12. Let $X=[0,1]$ be endowed with the usual metric $d(x, y)=|x-y|$ for all $x, y \in X$ so that $(X, d)$ is a complete metric space. Also, let $F: X \rightarrow 2^{X} \backslash\{\emptyset\}$ be defined by

$$
F x= \begin{cases}{\left[0, \frac{x}{2}\right]} & \text { if } x \in[0,1[, \\ {\left[\frac{1}{2}, 1\right]} & \text { if } x=1 .\end{cases}
$$

Consider $q=4 / 3, a=1 / 2$, and $b=1 / 6$ such that $a q+b=$ $5 / 6<1$. Then, for $x \neq 1$ and $y=x / 2$, we have $d(x, y)=$ $x / 2 \leq(4 / 3) \cdot(x / 2)=(4 / 3) D(x, F x)$; that is $y \in S_{q}(x)$. Moreover,

$$
\begin{aligned}
D(y, F y) & =D\left(\frac{x}{2},\left[0, \frac{x}{4}\right]\right)=\frac{x}{4} \leq \frac{1}{2} \cdot \frac{x}{2}+\frac{1}{6} \cdot \frac{x}{2} \\
& =a d(x, y)+b D(x, F x) .
\end{aligned}
$$

Also, for $x=1$ and $y=1$, we have $d(x, y)=0=q \cdot 0=$ $q D(x, F x)$; that is, $1 \in S_{q}(x)$. Moreover,

$$
D(y, F y)=D\left(1,\left[\frac{1}{2}, 1\right]\right)=0 \leq a d(x, y)+b D(x, F x) .
$$

Finally, the function $p: X \rightarrow[0,+\infty)$ defined by

$$
p x=D(x, F x)= \begin{cases}\frac{x}{2} & \text { if } x \in[0,1[, \\ 0 & \text { if } x=1\end{cases}
$$

is lower semicontinuous in $[0,1]$. Thus, all the hypotheses of Theorem 11 are satisfied and so $F$ has a selection $f$ that is Caristi's mapping. In fact, $f: X \rightarrow X$, defined by $f x=x / 2$ for all $x \in X$, is such that $f x \in F x$ and also $d(x, f x)=x / 2=$ $\phi(x)-\phi(f x)$, where $\phi: X \rightarrow[0,+\infty)$ is given by $\phi(x)=x$ for all $x \in X$.

Notice that

$$
H(F 0, F 1)=H\left(\{0\},\left[\frac{1}{2}, 1\right]\right)=\max \left\{\frac{1}{2}, 1\right\}=d(0,1)
$$

and hence $F$ is not Nadler's multivalued contraction.

Analogous results to Theorem 11 can be established under different hypotheses. For instance, in the next theorem, the multivalued mapping $F$ satisfies another contractive condition.

Theorem 13. Let $(X, d)$ be a complete metric space. Let $F$ : $X \rightarrow 2^{X} \backslash\{\emptyset\}$ be a multivalued mapping and let $q>1$ be a real number. Consider $S_{q}(x):=\{y \in F x: d(x, y) \leq q D d(x, F x)\}$ and suppose that $F$ satisfies the following conditions:

(i) there exist nonnegative real numbers $\alpha, \beta, \gamma$ with $(\alpha+$ $\gamma) q+\beta+\gamma<1$ such that, for each $x \in X$, there is $y \in S_{q}(x)$ having the property

$D(y, F y) \leq \alpha d(x, y)+\beta D(x, F x)+\gamma D(x, F y) ;$ (ii) the function $p: X \rightarrow[0,+\infty)$ defined by $p x:=$ $D(x, F x)$, for all $x \in X$, is lower semicontinuous.

Then $F$ has a selection $f$ that is Caristi's mapping.

Proof. By the axiom of choice and condition (i), there is a mapping $f: X \rightarrow X$ with $f x \in S_{q}(x)$ for all $x \in X$ such that

$$
D(f x, F f x) \leq \alpha d(x, f x)+\beta D(x, F x)+\gamma D(x, F f x)
$$

and so, by using the triangular inequality for $D(x, F f x)$,

$$
(1-\gamma) D(f x, F f x)-\beta D(x, F x) \leq(\alpha+\gamma) d(x, f x) .
$$

Then

$$
\begin{aligned}
(1- & \beta-\gamma-(\alpha+\gamma) q) d(x, f x) \\
\leq & (1-\beta-\gamma) q D(x, F x)-(\alpha+\gamma) q d(x, f x) \\
\leq & (1-\beta-\gamma) q D(x, F x)-(1-\gamma) q D(f x, F f x) \\
& +\beta q D(x, F x) \\
= & (1-\gamma) q D(x, F x)-(1-\gamma) q D(f x, F f x) .
\end{aligned}
$$

By condition (ii), the function $\phi: X \rightarrow[0,+\infty)$ defined by $\phi(t):=((1-\gamma) q /(1-\beta-\gamma-(\alpha+\gamma) q)) D(t, F t)$, for all $t \in X$, is lower semicontinuous and hence $f$ is Caristi's mapping that is a selection of $F$.

We would like to remark that other results can be stated by involving upper semicontinuous multivalued mappings, in view of the following situation.

Definition 14. Let $(X, d)$ be a metric space. Then, a multivalued mapping $F: X \rightarrow 2^{X} \backslash\{\emptyset\}$ is said to be $h$-upper semicontinuous at $x_{0} \in X$, if the function

$$
h\left(F x, F x_{0}\right):=\sup \left\{D\left(y, F x_{0}\right): y \in F x\right\}
$$

is continuous at $x_{0}$. Clearly, $F$ is said to be $h$-upper semicontinuous, whenever $h\left(F x, F x_{0}\right)$ is continuous at every $x_{0} \in X$.

Now we present a class of multivalued mappings such that the function $\phi(x)=D(x, F x)$, for all $x \in X$, is lower semicontinuous.

Proposition 15. Let $(X, d)$ be a metric space. If $F: X \rightarrow$ $2^{X} \backslash\{\emptyset\}$ is $h$-upper semicontinuous, then the function $\phi(x)=$ $D(x, F x)$ is lower semicontinuous.

Proof. Given $x \in X$, for all $y \in X$, we get

$$
\begin{aligned}
\phi(x) & =D(x, F x) \leq d(x, y)+D(y, F y)+h(F y, F x) \\
& \leq d(x, y)+\phi(y)+h(F y, F x) .
\end{aligned}
$$

From above inequalities, we deduce that $\phi(x) \leq$ $\liminf _{y \rightarrow x} \phi(y)$ and so $\phi$ is a lower semicontinuous function. 
For instance, from Theorem 13 and Proposition 15 we get the following corollary.

Corollary 16. Let $(X, d)$ be a complete metric space. Let $F$ : $X \rightarrow 2^{X} \backslash\{\emptyset\}$ be an $h$-upper semicontinuous multivalued mapping and let $q>1$ be a real number. Consider $S_{q}(x):=$ $\{y \in F x: d(x, y) \leq q D(x, F x)\}$ and suppose that $F$ satisfies the condition ( $i$ ) of Theorem 13. Then $F$ has a selection $f$ that is Caristi's mapping.

\section{Extension to Quasi-Fixed Point Theorems}

Let $(X, d)$ be a metric space. We recall that a multivalued mapping $F: X \rightarrow 2^{X} \backslash\{\emptyset\}$ has a quasi-fixed point if there exists a point $z \in X$ such that $D(z, F z)=0$. Then we extend our theory by considering functions instead of constant values. Therefore, let $(X, d)$ be a metric space, and let $a, b: X \rightarrow[0,+\infty)$ and $q: X \rightarrow(0,+\infty)$ be functions such that

$$
a(x) q^{-1}(x)+b(x)<1 \quad \forall x \in X
$$

Remark 17. Notice that in (22) we do not need that $q(x)<1$. We will return on this fact to derive a particular situation from the following theorem.

Theorem 18. Let $(X, d)$ be a complete metric space. Let $F$ : $X \rightarrow 2^{X} \backslash\{\emptyset\}$ be a multivalued mapping. Suppose that $F$ satisfies the following conditions:

(i) there exist three functions $a, b: X \rightarrow[0,+\infty)$ and $q: X \rightarrow(0,+\infty)$ such that (22) holds;

(ii) for each $x \in X$, with $D(x, F x)>0$, there exists $y \in$ $X \backslash\{x\}$ such that

$$
\begin{gathered}
q(x) d(x, y) \leq D(x, F x), \\
D(y, F y) \leq a(x) d(x, y)+b(x) D(x, F x) ;
\end{gathered}
$$

(iii) the function $p: X \rightarrow[0,+\infty)$ defined by $p x:=$ $D(x, F x)$, for all $x \in X$, is lower semicontinuous;

(iv) there exists $\eta>0$ such that

$$
\begin{array}{r}
\inf \{q(x)-b(x) q(x)-a(x): x \in X, \\
\left.D(x, F x) \leq \inf _{z \in X} D(z, F z)+\eta\right\}>0 .
\end{array}
$$

Then $F$ has a quasi-fixed point; that is, there exists $z \in X$ such that $D(z, F z)=0$.

Proof. Assume that $D(x, F x)>0$ for all $x \in X$. By the axiom of choice and condition (ii), there is a mapping $f: X \rightarrow X$ with $f x \neq x$ such that

$$
\begin{gathered}
q(x) d(x, f x) \leq D(x, F x), \\
D(f x, F f x) \leq a(x) d(x, f x)+b(x) D(x, F x) .
\end{gathered}
$$

Then for each $x \in X$

$$
D(f x, F f x)-b(x) D(x, F x) \leq a(x) d(x, f x) .
$$

This implies

$$
\begin{aligned}
(1 & \left.-b(x)-a(x) q^{-1}(x)\right) d(x, f x) \\
\leq & (1-b(x)) q^{-1}(x) D(x, F x)-a(x) q^{-1}(x) d(x, f x) \\
\leq & (1-b(x)) q^{-1}(x) D(x, F x)-q^{-1}(x) D(f x, F f x) \\
& +b(x) q^{-1}(x) D(x, F x) \\
= & q^{-1}(x) D(x, F x)-q^{-1}(x) D(f x, F f x) .
\end{aligned}
$$

Consequently, we have

$$
\begin{aligned}
d(x, f x) \leq & \frac{1}{q(x)-b(x) q(x)-a(x)} \\
& \times[D(x, F x)-D(f x, F f x)] .
\end{aligned}
$$

Now, let $Y:=\left\{x \in X: D(x, F x) \leq \inf _{z \in X} D(z, F z)+\eta\right\}$. Since, by (iii), $Y$ is a closed subset of $X$, we deduce that $Y$ is complete. Denote by $\gamma:=\inf \{q(x)-b(x) q(x)-a(x): x \in Y\}>0$. For all $x \in Y$, we get

$$
d(x, f x) \leq \frac{1}{\gamma}[D(x, F x)-D(f x, F f x)]=\phi(x)-\phi(f x)
$$

where the function $\phi: X \rightarrow[0,+\infty)$ is defined by $\phi(t):=$ $\gamma^{-1} D(t, F t)$, for all $t \in X$. Clearly, by condition (iii), the function $\phi$ is lower semicontinuous. From (29), we get that $f x \in Y$ whenever $x \in Y$ and hence $f: Y \rightarrow Y$ is Caristi's mapping. This implies that $f$ has a fixed point in $Y$, a contradiction since $f x \neq x$ for all $x \in X$. Hence there is $z \in X$ such that $D(z, F z)=0$.

As a consequence of Theorem 18, in the case that Fx is also closed, we obtain the following corollary.

Corollary 19. Let $(X, d)$ be a complete metric space. Let $F$ : $X \rightarrow 2^{X} \backslash\{\emptyset\}$ be a multivalued mapping such that $F x$ is closed. Suppose that $F$ satisfies the following conditions:

(i) there exist three functions $a, b: X \rightarrow[0,+\infty)$ and $q: X \rightarrow(0,+\infty)$ such that (22) holds;

(ii) for each $x \in X$, with $D(x, F x)>0$, there exists $y \in$ $X \backslash\{x\}$ such that

$$
\begin{gathered}
q(x) d(x, y) \leq D(x, F x), \\
D(y, F y) \leq a(x) d(x, y)+b(x) D(x, F x)
\end{gathered}
$$

(iii) the function $p: X \rightarrow[0,+\infty)$ defined by $p x:=$ $D(x, F x)$, for all $x \in X$, is lower semicontinuous; 
(iv) there exists $\eta>0$ such that

$$
\begin{array}{r}
\inf \{q(x)-b(x) q(x)-a(x): x \in X, \\
\left.D(x, F x) \leq \inf _{z \in X} D(z, F z)+\eta\right\}>0 .
\end{array}
$$

Then $F$ has a fixed point.

In view of Remark 17, by assuming $q(x)<1$, for all $x \in X$, on the same lines of the proof of Theorem 18, one can prove the following result.

Theorem 20. Let $(X, d)$ be a complete metric space. Let $F$ : $X \rightarrow 2^{X} \backslash\{\emptyset\}$ be a multivalued mapping. Suppose that $F$ satisfies the following conditions:

(i) there exist three functions $a, b: X \rightarrow[0,1)$ and $q:$ $X \rightarrow(0,1)$ such that (22) holds;

(ii) for each $x \in X$, with $D(x, F x)>0$, there exists $y \in F x$ such that

$$
\begin{gathered}
q(x) d(x, y) \leq D(x, F x), \\
D(y, F y) \leq a(x) d(x, y)+b(x) D(x, F x) ;
\end{gathered}
$$

(iii) the function $p: X \rightarrow[0,+\infty)$ defined by $p x:=$ $D(x, F x)$, for all $x \in X$, is lower semicontinuous;

(iv) there exists $\eta>0$ such that

$$
\begin{array}{r}
\inf \{q(x)-b(x) q(x)-a(x): x \in X, \\
\left.D(x, F x) \leq \inf _{z \in X} D(z, F z)+\eta\right\}>0 .
\end{array}
$$

Then $F$ has a selection $f$ that is Caristi's mapping on a closed subset of $X$.

Remark 21. If, in Theorems 18 and 20 and Corollary 19, we assume that the multivalued mapping $F$ is $h$-upper semicontinuous, then (iii) holds true. In this case, we can reformulate the statements of these results, requiring that $F$ satisfies only conditions (i), (ii), and (iv).

\section{Generalization of Caristi's Theorem}

We denote by $\Phi$ the set of all functions $\zeta:[0,+\infty) \rightarrow$ $[0,+\infty)$ such that there exist $\varepsilon>0$ and $c \in(0,1)$ satisfying $\delta_{\varepsilon}=\sup \zeta^{-1}([0, \varepsilon])<+\infty, \zeta(t) \geq c t$ for all $t \in\left[0, \delta_{\varepsilon}\right]$ and $\zeta(t)>\varepsilon$ for all $t>\delta_{\varepsilon}$.

Remark 22. Given a nondecreasing function $\zeta:[0,+\infty) \rightarrow$ $[0,+\infty)$ continuous at $t=0$ with $\zeta(0)=0$, consider the right lower Dini derivative of $\zeta$ at $t \in[0,+\infty)$; that is,

$$
\left[D_{+} \zeta\right](t)=\liminf _{s \rightarrow t^{+}} \frac{\zeta(s)-\zeta(t)}{s-t}
$$

Then $\zeta \in \Phi$ provided that $\left[D_{+} \zeta\right](0)>0$; see [8]. Also, each function $\zeta:[0,+\infty) \rightarrow[0,+\infty)$ that is nondecreasing, subadditive, and continuous at $t=0$ with $\zeta(0)=0$ belongs to $\Phi$.
Inspired by Khamsi [13] and Jachymski [8], we give two fixed point theorems. In particular our first theorem furnishes an alternative proof to Theorem 3 of [13] and the related Kirk's problem, without using order relations (see Section 3 in [13] for more details).

Theorem 23. Let $(X, d)$ be a complete metric space. Let $f$ : $X \rightarrow X$ be a mapping. Suppose that there exist a lower semicontinuous function $\phi: X \rightarrow[0,+\infty)$ and a function $\zeta \in \Phi$ such that

$$
\zeta(d(x, f x)) \leq \phi(x)-\phi(f x) \quad \forall x \in X
$$

Then $f$ has a fixed point in $X$.

Proof. Let $\varepsilon>0, c \in(0,1)$, and let $\delta_{\varepsilon}$ be as stated above. Let

$$
Y:=\left\{x \in X: \phi(x) \leq \inf _{z \in X} \phi(z)+\varepsilon\right\} .
$$

The set $Y$ is closed since $\phi$ is lower semicontinuous and hence complete. Now, from (35), we get that $f x \in Y$ whenever $x \in$ $Y$. Also

$$
\zeta(d(x, f x)) \leq \phi(x)-\phi(f x) \leq \phi(x)-\inf _{z \in X} \phi(z) \leq \varepsilon
$$

for all $x \in Y$; we obtain that $d(x, f x) \in\left[0, \delta_{\varepsilon}\right]$ whenever $x \in$ $Y$. Hence

$$
c d(x, f x) \leq \zeta(d(x, f x)) \leq \phi(x)-\phi(f x) ;
$$

that is,

$$
d(x, f x) \leq \frac{1}{c} \phi(x)-\frac{1}{c} \phi(f x) \quad \forall x \in Y .
$$

Since the function $(1 / c) \phi$ is lower semicontinuous, by Theorem 3, the mapping $f: Y \rightarrow Y$ has a fixed point in $Y$ and so in $X$.

Example 24. Let $X=\{-3,-1\} \cup[0,+\infty)$ be endowed with the usual metric $d(x, y)=|x-y|$ for all $x, y \in X$ so that $(X, d)$ is a complete metric space. Also, let $f: X \rightarrow X$ be defined by

$$
f x= \begin{cases}0 & \text { if } x \notin[1,3] \\ 1 & \text { if } x \in[1,3] .\end{cases}
$$

It follows that

$$
d(x, f x)= \begin{cases}|x| & \text { if } x \notin[1,3] \\ x-1 & \text { if } x \in[1,3] .\end{cases}
$$

Notice that $\phi: X \rightarrow[0,+\infty)$, defined by $\phi(x)=|x|$ for all $x \in X$, is a lower semicontinuous function such that $\zeta(d(x, f x)) \leq \phi(x)-\phi(f x)$, where $\zeta:[0,+\infty) \rightarrow[0,+\infty)$ is given by $\zeta(t)=c t$ for all $t \geq 0$, where $c \in(0,1)$. Thus, we can apply Theorem 23 to conclude that $f$ has a fixed point; clearly 0 and 1 are fixed points of $f$.

The inspiration of our next theorem is Theorem 10. In particular, our result does not use a monotonic condition. For a comprehensive discussion, we refer the reader to the fundamental paper of Jachymski [8]. 
Theorem 25. Let $(X, d)$ be a complete metric space. Let $F$ : $X \rightarrow \mathrm{Cl}(X)$ be a multivalued mapping. Suppose that $F$ is a $\mu$-contraction with $\mu$ right upper semicontinuous such that the function $\zeta(t):=(t-\mu(t)) / 2$ for all $t \geq 0$ belongs to $\Phi$. Then $F$ has a fixed point.

Proof. Let $v:[0,+\infty) \rightarrow[0,+\infty)$ be the function defined by $\nu(t):=(t+\mu(t)) / 2$, for all $t \geq 0$. Clearly, $v$ is right upper semicontinuous and $\nu(t)<t$ for all $t>0$. Therefore, the set

$$
\{y \in F x: \nu(d(x, y)) \leq D(x, F x)\} \neq \emptyset
$$

for all $x \in X$. In fact, if $\left\{y_{n}\right\} \subset F x$ is a sequence such that $d\left(x, y_{n}\right) \downarrow D(x, F x)$, the right upper semicontinuity of the function $v$ ensures that

$$
\limsup _{n \rightarrow+\infty} v\left(d\left(x, y_{n}\right)\right) \leq v(D(x, F x))
$$

and hence there exists $y_{n}$ such that

$$
v\left(d\left(x, y_{n}\right)\right)<D(x, F x) .
$$

The axiom of choice ensures that there is a mapping $f: X \rightarrow$ $X$ such that

$$
f x \in F x, \quad v(d(x, f x)) \leq D(x, F x) \quad \text { for } x \in X .
$$

Since $D(f x, F f x) \leq H(F x, F f x) \leq \mu(d(x, f x))$, we get

$$
\begin{aligned}
\zeta(d(x, f x)) & =\nu(d(x, f x))-\mu(d(x, f x)) \\
& \leq D(x, F x)-D(f x, F f x) \\
& =\phi(x)-\phi(f x),
\end{aligned}
$$

where the function $\phi: X \rightarrow[0,+\infty)$ is defined by $\phi(x):=D(x, F x)$, for all $x \in X$. Since the function $\phi$ is lower semicontinuous, then we get that $f$ has a fixed point, which is a fixed point for $F$.

\section{Conclusion}

Under suitable hypotheses for multivalued mappings, we established the existence of Caristi type selections. Also we proved fixed point and quasi-fixed point theorems, by using weaker and modified hypotheses on some classes of functions present in the literature. Our results extend and complement many theorems in the literature.

\section{Conflict of Interests}

The authors declare that there is no conflict of interests regarding the publication of this paper.

\section{Authors' Contribution}

All authors contributed equally and significantly in writing this paper. All authors read and approved the final paper.

\section{Acknowledgments}

The first author is a Member of the Gruppo Nazionale per l'Analisi Matematica, la Probabilità e le loro Applicazioni (GNAMPA) of the Istituto Nazionale di Alta Matematica (INdAM). The second author is a Member of the Gruppo Nazionale per le Strutture Algebriche, Geometriche e le loro Applicazioni (GNSAGA) of the Istituto Nazionale di Alta Matematica (INdAM).

\section{References}

[1] D. Repovs and P. V. Semenov, Continuous Selections of Multivalued Mappings, vol. 455 of Mathematics and Its Applications, 1998.

[2] E. Michael, "Continuous selections. I," Annals of Mathematics: Second Series, vol. 63, pp. 361-382, 1956.

[3] A. Petruşel, "Multivalued operators and continuous selections," Pure Mathematics and Applications, vol. 9, no. 1-2, pp. 165-170, 1998.

[4] A. Petruşel and G. Petruşel, "Selection theorems for multivalued generalized contractions," Mathematica Moravica, vol. 9, pp. 43-52, 2005.

[5] A. Sîntămărian, "Selections and common fixed points for some generalized multivalued contractions," Demonstratio Mathematica, vol. 39, no. 3, pp. 609-617, 2006.

[6] A. Petruşel and A. Sîntămărian, "Single-valued and multivalued Caristi type operators," Publicationes Mathematicae Debrecen, vol. 60, no. 1-2, pp. 167-177, 2002.

[7] F. E. Browder, "The fixed point theory of multi-valued mappings in topological vector spaces," Mathematische Annalen, vol. 177, pp. 283-301, 1968.

[8] J. R. Jachymski, "Caristi's fixed point theorem and selections of set-valued contractions," Journal of Mathematical Analysis and Applications, vol. 227, no. 1, pp. 55-67, 1998.

[9] J. Caristi, "Fixed point theorems for mappings satisfying inwardness conditions," Transactions of the American Mathematical Society, vol. 215, pp. 241-251, 1976.

[10] H. Covitz and S. B. Nadler Jr., "Multi-valued contraction mappings in generalized metric spaces," Israel Journal of Mathematics, vol. 8, pp. 5-11, 1970.

[11] S. B. Nadler Jr., "Multi-valued contraction mappings," Pacific Journal of Mathematics, vol. 30, pp. 475-488, 1969.

[12] H.-K. Xu, "Some recent results and problems for set-valued mappings," in Advances in Mathematics Research, vol. 1, pp. 3149, 2002.

[13] M. A. Khamsi, "Remarks on Caristi's fixed point theorem," Nonlinear Analysis. Theory, Methods \& Applications, vol. 71, no. 1-2, pp. 227-231, 2009. 


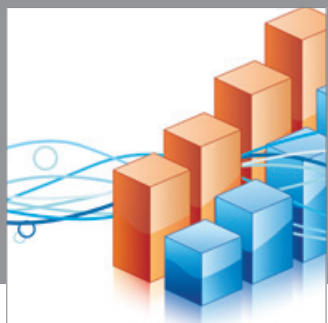

Advances in

Operations Research

mansans

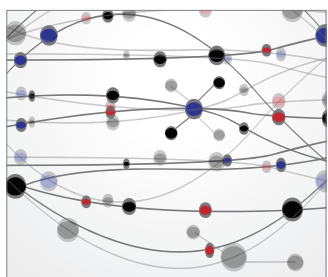

The Scientific World Journal
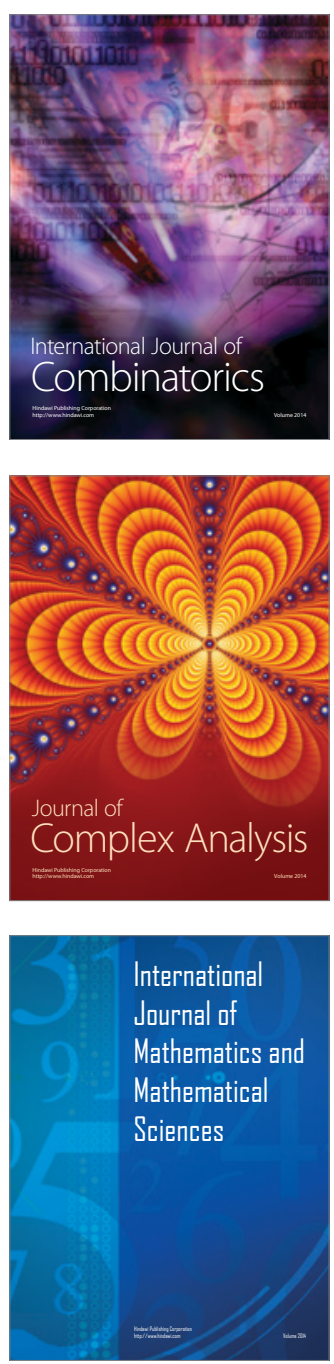
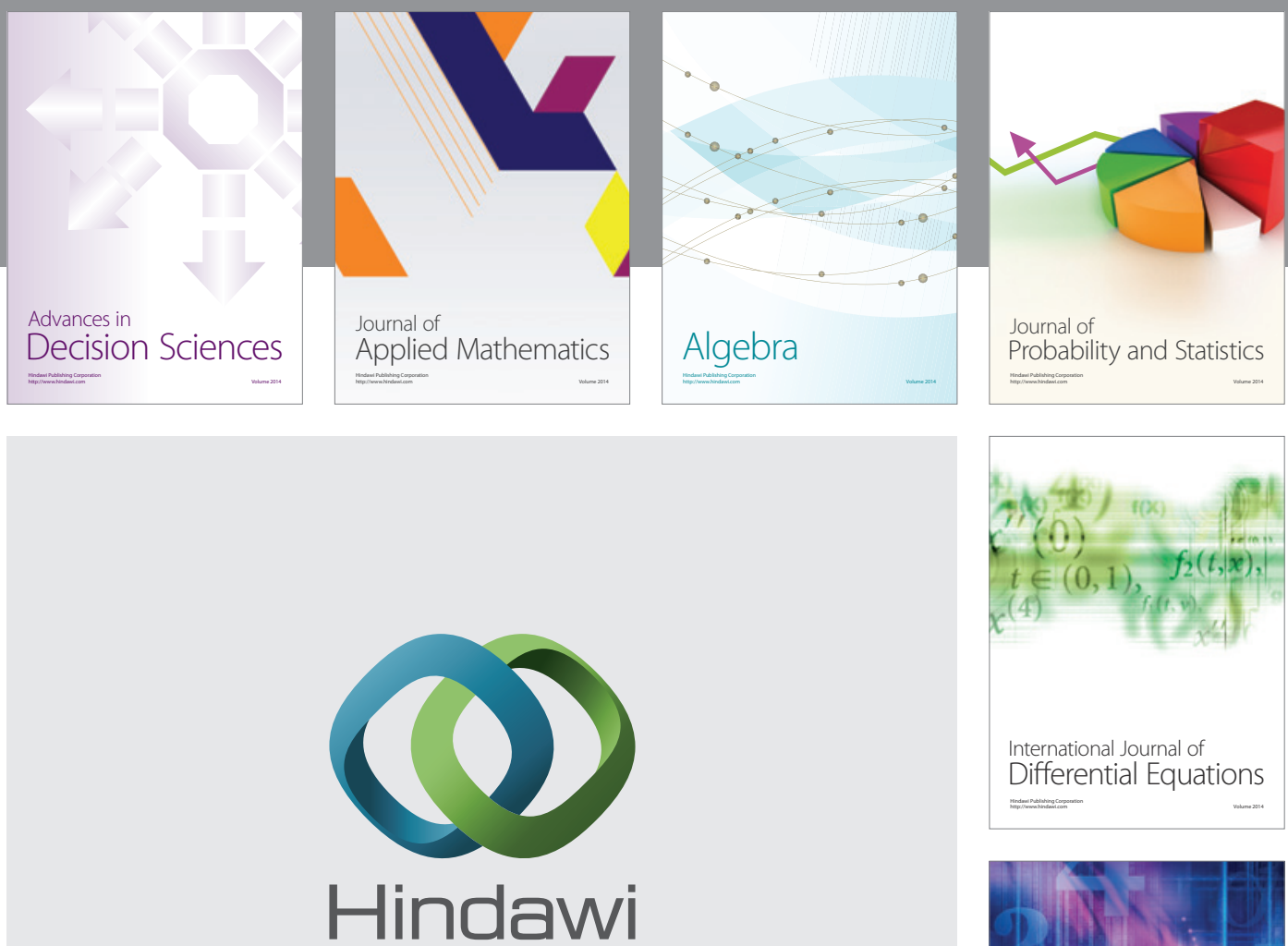

Submit your manuscripts at http://www.hindawi.com
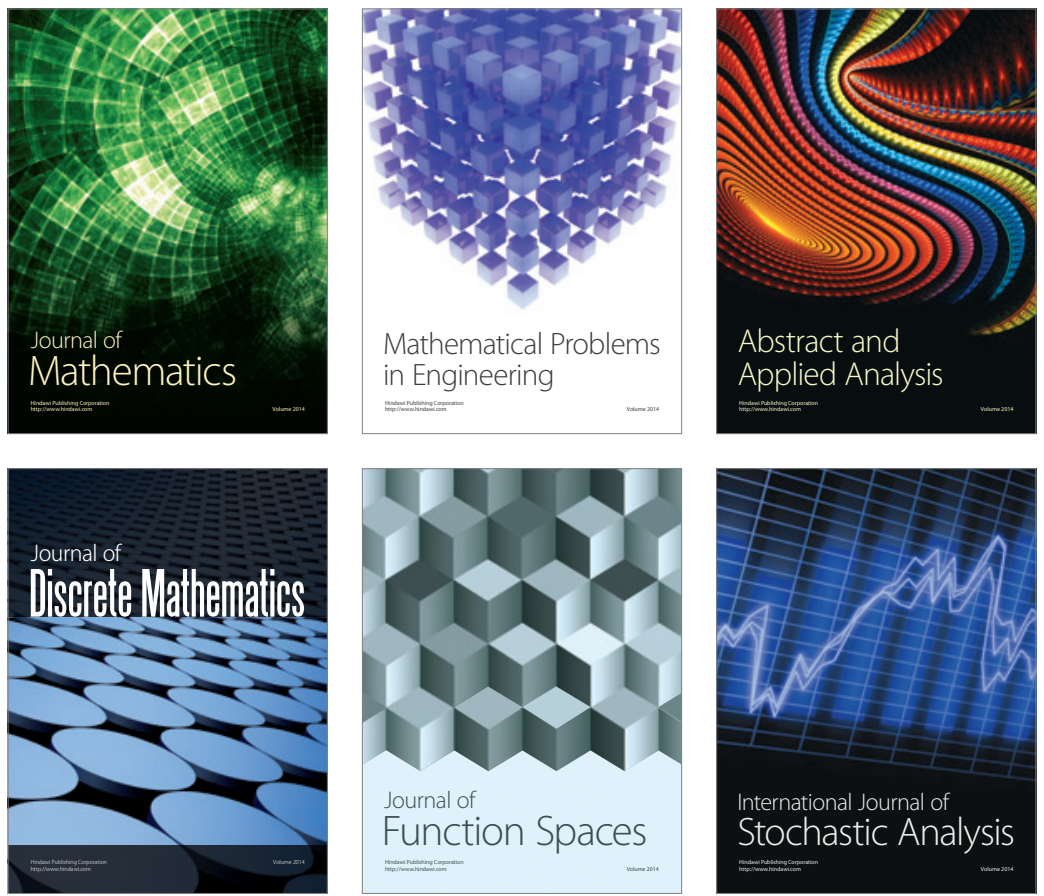

Journal of

Function Spaces

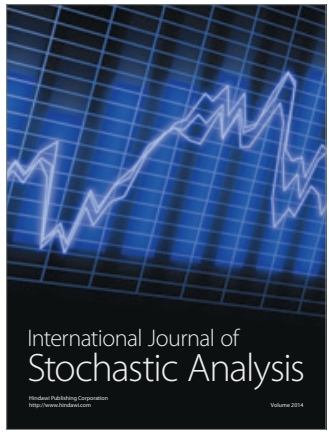

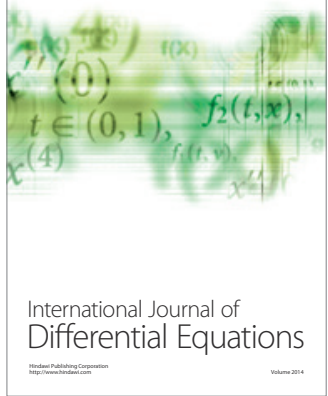
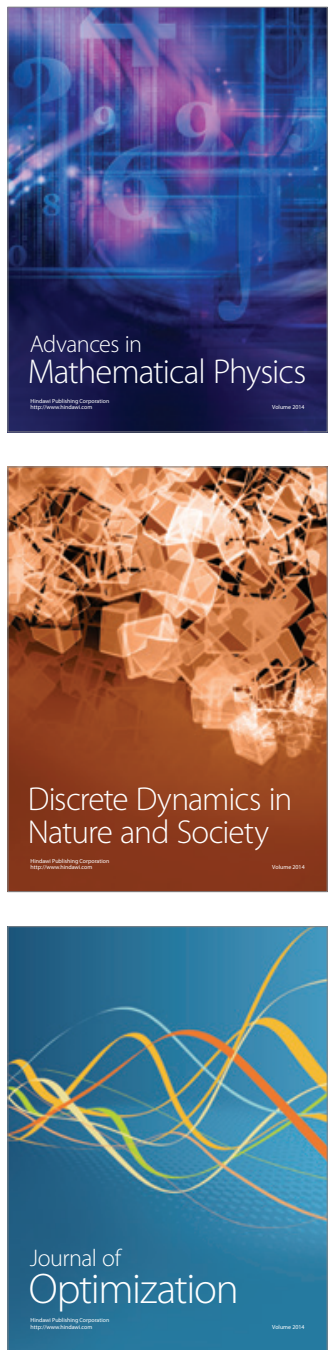